\title{
ISOLATED SINGULARITIES OF BINARY DIFFERENTIAL EQUATIONS OF DEGREE $n$
}

\author{
T. Fukui And J. J. Nuño-BAllesteros
}

\begin{abstract}
We study isolated singularities of binary differential equations of degree $n$ which are totally real. This means that at any regular point, the associated algebraic equation of degree $n$ has exactly $n$ different real roots (this generalizes the so called positive quadratic differential forms when $n=2$ ). We introduce the concept of index for isolated singularities and generalize Poincaré-Hopf theorem and Bendixson formula. Moreover, we give a classification of phase portraits of the $n$-web around a generic singular point. We show that there are only three types, which generalize the Darbouxian umbilics $D_{1}, D_{2}$ and $D_{3}$.
\end{abstract}

2010 Mathematics Subject Classification: Primary: 37C15; Secondary: 34C20, $34 \mathrm{~A} 34,53 \mathrm{~A} 07,53 \mathrm{~A} 60$.

Key words: totally real differential form, principal lines, Darbouxian umbilics, index.

\section{Introduction}

The study of the principal foliations near an isolated umbilic point of a surface $M$ immersed in $\mathbb{R}^{3}$ leads us to the consideration of quadratic binary differential equations (BDE) of the form

$$
a(x, y) d x^{2}+2 b(x, y) d x d y+c(x, y) d y^{2}=0,
$$

where $a(x, y), b(x, y), c(x, y)$ are smooth functions in some open subset $U \subset \mathbb{R}^{2}$ which are defined, after taking a parametrization of $M$, by means of the coefficients of the first and second fundamental form of $M$. Since the principal lines are orthogonal in the induced metric of $M$, the discriminant is $\Delta=b(x, y)^{2}-a(x, y) c(x, y) \geq 0$, with equality if and only if $(x, y)$ corresponds to an umbilic of $M$, so that $a(x, y)=b(x, y)=c(x, y)=0$ and hence, $(x, y)$ is a singularity of the BDE. It was Darboux [5] who considered the generic singularities and 
discovered there are only three topological types, known as the Darbouxian umbilics $D_{1}, D_{2}$ and $D_{3}$ (see $[\mathbf{1}]$ and $[\mathbf{1 9}]$ for a modern and precise study of this classification).

In fact, we can consider quadratic BDE of this type for general functions $a(x, y), b(x, y)$ and $c(x, y)$, with the discriminant having the property: $\Delta \geq 0$ with equality if and only if $a(x, y)=b(x, y)=c(x, y)=0$. The quadratic forms with this property are called positive and have been studied by many authors $[\mathbf{2}],[\mathbf{6}],[\mathbf{1 1}],[\mathbf{1 3}],[\mathbf{1 4}]$. A positive quadratic differential form defines a pair of transverse foliations in the region of regular points. Moreover, Guíñez showed that in this more general situation, the only generic singularities are again the Darbouxian umbilics $D_{1}$, $D_{2}$ and $D_{3}$.

The aim of this paper is to generalize this to degree $n$ BDE of the form

$$
a_{0}(x, y) d x^{n}+a_{1}(x, y) d x^{n-1} d y+\cdots+a_{n}(x, y) d y^{n}=0
$$

where $a_{i}(x, y)$ are smooth functions defined on $U \subset \mathbb{R}^{2}$ such that for any $(x, y) \in U$, either it is a singular point (that is, $a_{i}(x, y)=0$ for any $i=1, \ldots, n)$ or the associated algebraic equation has exactly $n$ different real roots. If the functions $a_{i}(x, y)$ have this property, then we say that the associated binary differential $n$-form $\omega=\sum_{i=1}^{n} a_{i}(x, y) d x^{n-i} d y^{i}$ is totally real.

When $n=1$, a differential form is always totally real and it induces an oriented foliation in the plane with singularities. For $n=2$, totally real is equivalent to positive in the Guínez sense and hence, the BDE defines a pair of transverse (non oriented) foliations. However, for $n \geq 3$, the corresponding BDE induces locally an $n$-web in the regular region (that is, a set of $n$ foliations $\left\{\mathcal{F}_{1}, \ldots, \mathcal{F}_{n}\right\}$ which are pairwise transverse). It seems that isolated singularities of $n$-webs in the plane have not been considered previously in the literature. Moreover, we feel that the use of degree $n$ BDE is a good approach to treat this subject.

The topological configuration of an $n$-web $(n \geq 3)$ can be extremely complicated, even in the regular case. When $n=3$, the curvature of the web is a 2 -form which is a topological invariant. Hence, even for regular webs we find that the topological classification has functional moduli. It is known that a regular 3 -web is parallelizable or hexagonal (that is, equivalent to three families of parallel straight lines) if and only if the curvature is zero. We should also mention that because of the rigidity of webs (any homeomorphism between two regular webs is in fact a diffeomorphism $[\mathbf{7}]$ ) the topological and differentiable classifications are the same. 
We show here that for $n \geq 3$, the classification of generic singularities of totally real differential $n$-forms gives again only three types, which we call the generalized Darbouxian $D_{1}, D_{2}$ and $D_{3}$. Here, generic means a generic choice of coefficients in the linear part of the functions $a_{i}(x, y)$. Moreover, the classification has to be understood not as a topological classification, but just as a description of the phase portrait of the foliations around the singular point.

One of the main ingredients of the classification is the index of an isolated singular point. It is defined as a rational number of the form $k / n$, where $k \in \mathbb{Z}$ and it can be interpreted as the rotation number of a continuously chosen vector tangent to the leaves, when we make a trip around the singular point. We also show the generalization of the Poincaré-Hopf theorem: if $M$ is a compact surface and $\omega$ is a totally real $n$-form with a finite number of singular points, then the sum of the indices is equal to the Euler characteristic $\chi(M)$.

Another important point in the paper is the use of complex coordinates. By setting $z=x+i y$ and $\bar{z}=x-i y$, we can express any $n$-form as $\omega=A_{0} d z^{n}+A_{1} d z^{n-1} d \bar{z}+\cdots+A_{n} d \bar{z}^{n}$, where $A_{j}=\bar{A}_{n-j}$ are differentiable functions. Then the index of an isolated singular point is equal to $-\operatorname{deg}\left(A_{0}\right) / n$, where $\operatorname{deg}\left(A_{0}\right)$ is the mapping degree of $A_{0}$. This implies that generically, the index is $\pm 1 / n$.

The final ingredient for the classification is the use of the polar blowup method to study singularities with a non degenerate principal part (see $[\mathbf{3}]$ and $[\mathbf{1 3}]$ for related results for vector fields or quadratic forms). We obtain a generalization of the Bendixson formula, which says that the index is equal to $1+(e-h) / 2 n$ where $e, h$ are the number of elliptic and hyperbolic sectors respectively. For a non degenerate singularity, the blow-up produces an $n$-form which has only singularities of saddle/node type. The configuration of these singularities gives a description of the phase portrait of the foliations around the singular point.

The original motivation of the authors to study singularities of differential $n$-forms comes from the study of higher order principal lines and umbilics of surfaces $M$ immersed in some Euclidean space $\mathbb{R}^{N}$. The obtained results will appear in a forthcoming paper [10]. Other geometrical motivations of the same kind can be found also in the literature. For instance, in [16] Little studies several BDEs associated to surfaces immersed in $\mathbb{R}^{4}$. Also in $[\mathbf{1 2}]$ the authors consider curvature lines of surfaces in $\mathbb{R}^{4}$ (in some sense) which are defined by a BDE of degree 4 .

Other nice results restricted to equiangular BDEs can be found in $[\mathbf{9}]$. We say that a totally real BDE is equiangular if at any regular point, the $n$ tangent directions to the foliations form a constant angle equal to $\pi / n$. 
If $n=2$, this means that the foliations are orthogonal. In general, the study of equiangular BDEs is equivalent to the study of cross fields in the terminology of Little [16].

\section{Totally real binary differential forms}

Definition 2.1. Let $M$ be a $C^{\infty}$ surface, i.e., a 2-dimensional manifold without boundary. A binary differential n-form on $M$ is a differentiable section of the symmetric tensor fiber bundle $S^{n}\left(T^{*} M\right)$. If we take coordinates $x, y$ on some open subset $U \subset M$, any binary differential $n$-form can be written in a unique way as

$$
\omega=\sum_{i=0}^{n} f_{i} d x^{i} d y^{n-i},
$$

where $f_{i}: U \rightarrow \mathbb{R}$ are smooth functions.

Given $p \in M, \omega(p): T_{p} M \rightarrow \mathbb{R}$ is a binary form of degree $n$. We say that $\omega$ is totally real at $p$ if either $\omega(p)=0$ or there are $n$ linear forms $\lambda_{1}, \ldots, \lambda_{n} \in T_{p} M^{*}$ which are pairwise linearly independent and such that $\omega(p)=\lambda_{1} \ldots \lambda_{n}$. We say that $\omega$ is totally real if it is totally real at any point of $M$.

Assume $\omega$ is totally real. A point $p \in M$ is called singular if $\omega(p)=0$ and regular otherwise. We will denote by $\operatorname{Sing}(\omega)$ the set of singular points of $\omega$. (In general, if the form is not totally real a singular point is usually considered when the linear forms are not pairwise linearly independent.)

A linear differential form $(n=1)$ is always totally real. In the case $n=$ 2 , a quadratic differential form is totally real if it is positive in the sense of [11]. Take local coordinates $x, y$ defined on some open subset $U \subset M$ and assume that $\omega$ is given by

$$
\omega=A d x^{2}+2 B d x d y+C d y^{2},
$$

for some smooth functions $A, B, C: U \rightarrow \mathbb{R}$. Then $\omega$ is totally real in $U$ if and only if for any $p \in U$, either $A(p)=B(p)=C(p)=0$ or $B^{2}(p)-A(p) C(p)>0$.

Definition 2.2. A (1-dimensional) $n$-web on a surface $M$ is a smooth choice of $n$ pairwise transverse tangent lines in $T_{p} M$ for each point $p \in$ $M$. In particular, in a neighbourhood $U$ of $p$, there are $n$ (1-dimensional) foliations $\left\{\mathcal{F}_{1}, \ldots, \mathcal{F}_{n}\right\}$ on $U$ such that they are pairwise transverse at any point and which correspond to the integral curves of the tangent lines. 
Observe that, in general, it is not possible to extend the $n$ foliations $\left\{\mathcal{F}_{1}, \ldots, \mathcal{F}_{n}\right\}$ to global foliations on $M$ (unless it is simply connected) since we may have monodromy between the foliations.

If $\omega$ is a totally real differential $n$-form on $M$, then we can associate an $n$-web on $M \backslash \operatorname{Sing}(\omega)$ in the following way. For each $p \in M \backslash \operatorname{Sing}(\omega)$, there are pairwise linearly independent linear forms $\lambda_{1}, \ldots, \lambda_{n} \in T_{p} M^{*}$ such that $\omega(p)=\lambda_{1} \ldots \lambda_{n}$. Moreover, it is possible to choose these linear forms so that they depend smoothly on $p$ (and hence define differential linear forms) on some open neighbourhood $U \subset M$. Then, the $n$-web is just defined by taking $\mathcal{F}_{i}$ as the foliation determined by $\lambda_{i}$ on $U$ (that is, the tangent vectors to $\mathcal{F}_{i}$ are the null vectors of $\lambda_{i}$ ).

Two totally real differential $n$-forms $\omega_{1}$ and $\omega_{2}$ define the same $n$-web on $U$ if and only if there is a non-zero smooth function $f: U \rightarrow \mathbb{R}$ such that $\omega_{1}=f \omega_{2}$ on $U$.

Recall that if $\omega$ is a differential $n$-form on $N$ and $f: M \rightarrow N$ is a differentiable map between surfaces, then $f^{*} \omega$ is the $n$-form on $M$ given by $f^{*} \omega(p)(X)=\omega(f(p))\left(f_{*} X\right)$ for any $p \in M$ and $X \in T_{p} M$, where $f_{*}: T_{p} M \rightarrow T_{f(p)} N$ the differential of $f$ at the point $p$.

Definition 2.3. Let $\omega_{1}, \omega_{2}$ be two totally real differential $n$-forms defined on surfaces $M, N$ respectively. We say that they are $C^{\infty}$-equivalent (resp. topologically equivalent) if there is a $C^{\infty}$ diffeomorphism (resp. homeomorphism) $\phi: M \rightarrow N$ such that

(1) $\phi\left(\operatorname{Sing}\left(\omega_{1}\right)\right)=\operatorname{Sing}\left(\omega_{2}\right)$,

(2) $\phi: M \backslash \operatorname{Sing}\left(\omega_{1}\right) \rightarrow N \backslash \operatorname{Sing}\left(\omega_{2}\right)$ preserves the leaves of the foliations of the $n$-webs defined by $\omega_{1}, \omega_{2}$.

If $\phi$ is a $C^{\infty}$ diffeomorphism, then condition (2) is equivalent to the existence of a nonzero smooth function $f: M \backslash \operatorname{Sing}\left(\omega_{1}\right) \rightarrow \mathbb{R}$ such that $\phi^{*}\left(\omega_{2}\right)=f \omega_{1}$ on $M \backslash \operatorname{Sing}\left(\omega_{1}\right)$.

\section{The index of an isolated singular point}

We will define an index for isolated singular points of totally real differential forms, which generalize the index in the case of linear or quadratic forms.

Let $\omega$ be a totally real differential $n$-form on a surface $M$ and $p \in M$ an isolated singular point. Assume that $M$ is orientable and choose an orientation. Moreover, we choose a Riemannian metric $g$ on $M$ and orthogonal coordinates $x, y$ on some open neighbourhood $U$ of $p$ in $M$, compatible with the orientation. Now, let $\alpha:[0, \ell] \rightarrow M$ be a simple, 
closed and piecewise regular curve, such that $\alpha([0, \ell]) \subset U$ is the boundary of a simple region $R$, which contains $p$ as the only singular point in the interior. Moreover, we assume that $\alpha$ goes through the boundary of $R$ in positive sense. Since $\alpha$ is a closed curve, we can extend it to $\alpha: \mathbb{R} \rightarrow M$, by setting $\alpha(t+\ell)=\alpha(t)$.

For each $t \in \mathbb{R}$ we choose a unit tangent vector $X(t)$ which is a solution of the equation $\omega(\alpha(t))(X)=0$ at the point $\alpha(t)$. Since it is an algebraic equation of degree $n$, we can choose $X(t)$ so that it defines a piecewise differentiable unit vector field along $\alpha$.

If we start with $t=0$, after a complete turn, $X(\ell)$ must coincide with one of the $2 n$ unit vectors which are solution of $\omega(\alpha(0))(X)=0$. Because of transversality, after $2 n$ turns in positive sense, we must return to the initial vector, that is, $X(2 n \ell)=X(0)$. Now, let $\theta(t)$ be a differentiable determination of the angle between $\left.\frac{\partial}{\partial x}\right|_{\alpha(t)}$ and $X(t)$. Then, $\theta(2 n \ell)$ and $\theta(0)$ differ by an integer multiple of $2 \pi$.

Definition 3.1. With the above notation, we define the index of $\omega$ in $p$ by

$$
\operatorname{ind}(\omega, p)=\frac{\theta(2 n \ell)-\theta(0)}{4 \pi n} .
$$

It follows from the definition that the index is always a rational number of the form $s / 2 n$, with $s \in \mathbb{Z}$.

The proof of the following lemma is standard and will be omitted.

Lemma 3.2. The index ind $(\omega, p)$ does not depend on the choice of:

(1) the determination of the angle $\theta$,

(2) the vector field $X$,

(3) the coordinates $x, y$,

(4) the curve $\alpha$,

(5) the Riemannian metric $g$,

(6) the orientation of $M$.

As a consequence of Lemma 3.2, we deduce that the index is well defined and it only depends on the differential form $\omega$. Moreover, the definition can be extended to the case that $M$ is not orientable by taking a local orientation in a neighbourhood of the singular point.

The definition of index can also be extended to the case where $p$ is a regular point, although in such case the index is always zero. In fact, we can take coordinates in such a way that $\partial / \partial x$ coincides with $X$ along $\alpha$ and hence, $\theta(t) \equiv 0$. 
Finally, another immediate consequence of Lemma 3.2 is that the index is invariant under $C^{\infty}$-equivalence. Let $\omega_{1}, \omega_{2}$ be two totally real differential $n$-forms defined on surfaces $M, N$ respectively, which are equivalent through the diffeomorphism $\phi: M \rightarrow N$. Then, for each $p \in \operatorname{Sing}\left(\omega_{1}\right)$,

$$
\operatorname{ind}\left(\omega_{1}, p\right)=\operatorname{ind}\left(\omega_{2}, \phi(p)\right) .
$$

Remark 3.3. We give here a formula which can be very useful to compute the index. Let us denote by $X_{1}(t), \ldots, X_{2 n}(t)$ the unit vector fields along $\alpha$ which are solution of $\omega(\alpha(t))(X)=0$. We assume that they are ordered so that

$$
\theta_{1}(t)<\theta_{2}(t)<\cdots<\theta_{2 n}(t)<\theta_{1}(t)+2 \pi,
$$

where $\theta_{j}(t)$ denotes the determination of the angle of each vector field $X_{j}(t)$. Since $X_{1}(\ell)=X_{i}(0)$ for some $i \in\{1, \ldots, 2 n\}$, there exists $m_{i} \in \mathbb{Z}$ such that

$$
\theta_{1}(\ell)=\theta_{i}(0)+2 \pi m_{i}
$$

We introduce the notation $\theta_{2 n+1}(t)=\theta_{1}(t)+2 \pi, \theta_{2 n+2}(t)=\theta_{2}(t)+2 \pi$, and in general, $\theta_{2 q n+j}(t)=\theta_{j}(t)+2 q \pi$, for any $q \in \mathbb{Z}$ and $j \in\{1, \ldots, 2 n\}$. Then,

$$
\begin{aligned}
\theta_{1}(\ell) & =\theta_{i}(0)+2 \pi m_{i}, \\
\theta_{1}(2 \ell) & =\theta_{i}(\ell)+2 \pi m_{i}=\theta_{2 i-1}(0)+4 \pi m_{i}, \\
& \cdots \\
\theta_{1}(2 n \ell) & =\theta_{2 n(i-1)+1}(0)+4 \pi n m_{i}=\theta_{1}(0)+2 \pi\left(2 n m_{i}+i-1\right) .
\end{aligned}
$$

From this, we arrive to

$$
\operatorname{ind}(\omega, p)=\frac{\theta_{1}(2 n \ell)-\theta_{1}(0)}{4 \pi n}=m_{i}+\frac{i-1}{2 n} .
$$

We finish this section by proving a generalization of the well known Poincaré-Hopf Theorem for vector fields or quadratic differential forms $[15],[4]$.

Theorem 3.4. Let $M$ a compact surface and let $\omega$ be a totally real differential $n$-form with a finite number of singular points $p_{1}, \ldots, p_{m}$. Then,

$$
\chi(M)=\sum_{i=1}^{m} \operatorname{ind}\left(\omega, p_{i}\right),
$$

where $\chi(M)$ denotes the Euler-Poincaré characteristic of $M$. 
Proof: The proof given here is just an adaptation of the proof given in $[\mathbf{4}$, p. 279] for the case of vector fields. We prove first the theorem in the case where $M$ is orientable.

We choose some orientation and a Riemannian metric on $M$. We take a triangulation $\mathcal{T}$ such that each triangle $T \in \mathcal{T}$ contains at most one singular point $p_{T}$ on the interior. Moreover, for each $T \in \mathcal{T}, X_{T}$ is a vector field along the boundary of $T$ which is a solution of equation $\omega(X)=0$. Then,

$$
\int_{\mathcal{T}} K d \sigma-2 \pi \operatorname{ind}\left(\omega, p_{T}\right)=\frac{\Delta_{T}}{2 n},
$$

where $\Delta_{T}$ denotes the variation of the angle from $X_{T}$ to some parallel vector field after going through the boundary of $T 2 n$ times in positive sense.

Now, summing up over $T \in \mathcal{T}$ and taking into account that each edge is common to two triangles with opposite orientations, we arrive to

$$
\int_{M} K d \sigma-2 \pi \sum_{T \in \mathcal{T}} \operatorname{ind}\left(\omega, p_{T}\right)=\sum_{T \in \mathcal{T}} \frac{\Delta_{T}}{2 n}=0 .
$$

Finally, the result is a consequence of the Gauss-Bonnet Theorem:

$$
\int_{M} K d \sigma=2 \pi \chi(M)
$$

When the surface $M$ is not orientable, we consider $\pi: \tilde{M} \rightarrow M$ a double covering, where $\tilde{M}$ is an orientable and compact surface.

\section{Differential forms in complex coordinates}

We identify $\mathbb{R}^{2}$ with $\mathbb{C}$ and use the following notation

$$
\begin{aligned}
z & =x+i y, & \bar{z} & =x-i y, \\
d z & =d x+i d y, & d \bar{z} & =d x-i d y, \\
\frac{\partial}{\partial z} & =\frac{1}{2}\left(\frac{\partial}{\partial x}-i \frac{\partial}{\partial y}\right), & \frac{\partial}{\partial \bar{z}} & =\frac{1}{2}\left(\frac{\partial}{\partial x}+i \frac{\partial}{\partial y}\right) .
\end{aligned}
$$

With this notation, any differential $n$-form on an open subset $U \subset \mathbb{C}$ can be written in a unique way in this coordinates as

$$
\omega=A_{0} d z^{n}+A_{1} d z^{n-1} d \bar{z}+\cdots+A_{n} d \bar{z}^{n},
$$

for some differentiable functions $A_{j}: U \rightarrow \mathbb{C}$ such that $A_{j}=\bar{A}_{n-j}$ for all $j=0, \ldots, n$.

The following theorem is a generalization of the result in [15, VII.2.3] in the case $n=2$. 
Theorem 4.1. Let $\omega$ be a totally real differential $n$-form on an open subset $U \subset \mathbb{C}$ and let $p \in U$ be an isolated singular point. Then, $p$ is an isolated zero of $A_{0}$ and

$$
\operatorname{ind}(\omega, p)=-\frac{\operatorname{deg}\left(A_{0}, p\right)}{n},
$$

where $\operatorname{deg}\left(A_{0}, p\right)$ denotes the local degree of $A_{0}$ at $p$.

Proof: Let $\delta>0$ small enough and let $\alpha(t)=p+\delta e^{i t}$, for $t \in \mathbb{R}$. We denote by $X_{1}(t), \ldots, X_{n}(t)$ unit vector fields along $\alpha$ which are pairwise linearly independent and are solution of the equation $\omega(\alpha(t))(X)=0$. We also denote by $\theta_{j}(t)$ a differentiable determination of the angle of $X_{j}(t)$, so that

$$
X_{j}(t)=e^{i \theta_{j}(t)} \frac{\partial}{\partial z}+e^{-i \theta_{j}(t)} \frac{\partial}{\partial \bar{z}} .
$$

Then $X_{j}(t)$ annihilates the linear form $\lambda_{j}(t)$ along $\alpha$ given by

$$
\lambda_{j}(t)=e^{i \phi_{j}(t)} d z+e^{-i \phi_{j}(t)} d \bar{z},
$$

where $\phi_{j}(t)=\pi / 2-\theta_{j}(t)$. Thus, by using elementary properties of the algebraic equations of degree $n$, we deduce that along $\alpha$ it is possible to factor $\omega$ as

$$
\omega(\alpha(t))=f(t) \lambda_{1}(t) \ldots \lambda_{n}(t),
$$

for some non vanishing function $f: \mathbb{R} \rightarrow \mathbb{R}$.

By comparing the coefficient of $d z^{n}$ in the above expression, we have

$$
A_{0}(\alpha(t))=f(t) e^{i\left(\phi_{1}(t)+\cdots+\phi_{n}(t)\right)} .
$$

Therefore $A_{0}(\alpha(t)) \neq 0$, for all $t \in \mathbb{R}$, which proves the first statement. Moreover, a differentiable determination of the angle of $A_{0}(\alpha(t))$ is given by

$$
\beta(t)=\phi_{1}(t)+\cdots+\phi_{n}(t)+\pi q,
$$

for some $q \in \mathbb{Z}$.

Finally,

$$
\begin{aligned}
\operatorname{deg}\left(A_{0}, p\right) & =\frac{\beta(4 \pi n)-\beta(0)}{4 \pi n}=\sum_{j=1}^{n} \frac{\phi_{j}(4 \pi n)-\phi_{j}(0)}{4 \pi n} \\
& =-\sum_{j=1}^{n} \frac{\theta_{j}(4 \pi n)-\theta_{j}(0)}{4 \pi n}=-n \operatorname{ind}(\omega, p) .
\end{aligned}
$$


Corollary 4.2. The index of any isolated singular point of a totally real differential $n$-form on a surface $M$ has the form $s / n$, with $s \in \mathbb{Z}$. Moreover, for each $s \in \mathbb{Z}$ there is a totally real differential $n$-form with an isolated singular point of index $s / n$.

Proof: The first part is an immediate consequence of Theorem 4.1. To see the second part, we take $M=\mathbb{C}, p=0$ and

$$
\omega= \begin{cases}z^{s} d z^{n}+\bar{z}^{s} d \bar{z}^{n}, & \text { if } s \geq 0, \\ \bar{z}^{|s|} d z^{n}+z^{|s|} d \bar{z}^{n}, & \text { if } s<0 .\end{cases}
$$

The above corollary says that the index of an isolated singular point of a totally real differential $n$-form is, in fact, of the form $s / n$, with $s \in \mathbb{Z}$, instead of $s / 2 n$. This can be interpreted as some kind of orientability condition for the $n$-web defined by the differential $n$-form.

For instance, when $n=1$, a linear differential form in $M$ induces an orientable foliation in a neighbourhood of each point of $M$. In this case, the index of an isolated singular point is an integer. However, the index of a (non necessarily orientable) foliation at an isolated singular point is in general a half-integer (see [15, VII.2.2]).

Corollary 4.3. Let $\omega$ be a totally real differential $n$-form on a surface $M$ and $p \in M$ an isolated singular point. Let $\alpha:[0, \ell] \rightarrow M$ be a curve satisfying the conditions in the definition of the index and let $X(t)$ be a unit vector field along $\alpha$, solution of $\omega(\alpha(t))(X)=0$. Then $X(n \ell)=$ $X(0)$ and

$$
\operatorname{ind}(\omega, p)=\frac{\theta(n \ell)-\theta(0)}{2 \pi n},
$$

where $\theta(t)$ denotes a determination of the angle of $X(t)$.

Proof: This is consequence of Corollary 4.2 and Remark 3.3. Let $X_{1}(t), \ldots, X_{2 n}(t)$ be the unit vector fields along $\alpha$ which are solution of $\omega(\alpha(t))(X)=0$, with $X(t)=X_{1}(t)$. We suppose that they are ordered so that

$$
\theta_{1}(t)<\theta_{2}(t)<\cdots<\theta_{2 n}(t)<\theta_{1}(t)+2 \pi,
$$

where $\theta_{j}(t)$ is the determination of the angle of each vector field $X_{j}(t)$. Then,

$$
\operatorname{ind}(\omega, p)=m_{i}+\frac{i-1}{2 n}
$$

where $\theta_{1}(\ell)=\theta_{i}(0)+2 \pi m_{i}$, with $m_{i} \in \mathbb{Z}$ and $i \in\{1, \ldots, 2 n\}$. Moreover, we introduce the notation $\theta_{2 q n+j}(t)=\theta_{j}(t)+2 q \pi$, for any $q \in \mathbb{Z}$ and $j \in\{1, \ldots, 2 n\}$. 
From Corollary 4.2 we see that $i-1$ must be even and hence, we can write $i-1=2 q$, with $q \in \mathbb{Z}$. Thus,

$$
\theta_{1}(n \ell)=\theta_{n(i-1)+1}(0)+2 \pi m_{i} n=\theta_{1}(0)+2 \pi\left(m_{i} n+q\right),
$$

giving $X_{1}(n \ell)=X_{1}(0)$.

Definition 4.4. We say that a singular point $p$ of a totally real differential $n$-form $\omega$ is simple if the linear part of $\omega$ at $p$ is itself a totally real differential $n$-form having $p$ as an isolated singular point. Suppose that in complex coordinates

$$
\omega=A_{0} d z^{n}+A_{1} d z^{n-1} d \bar{z}+\cdots+A_{n} d \bar{z}^{n},
$$

for some differentiable functions $A_{i}: U \rightarrow \mathbb{C}$. We also assume, for simplicity, that $p=0$. Then, each one of these functions $A_{i}$ has a Taylor expansion at the origin in the form

$$
A_{i}=a_{i} z+b_{i} \bar{z}+\cdots
$$

with $a_{i}, b_{i} \in \mathbb{C}$. The linear part of $\omega$ at $p$ is the differential $n$-form

$$
\omega_{1}=\left(a_{0} z+b_{0} \bar{z}\right) d z^{n}+\left(a_{1} z+b_{1} \bar{z}\right) d z^{n-1} d \bar{z}+\cdots+\left(a_{n} z+b_{n} \bar{z}\right) d \bar{z}^{n} .
$$

Corollary 4.5. Any simple singular point of a totally real differential $n$-form on a surface $M$ has index $\pm 1 / n$.

Proof: We take complex coordinates, suppose that $p=0$ and write the linear part of $\omega$ at $p$ in the form

$$
\omega_{1}=\left(a_{0} z+b_{0} \bar{z}\right) d z^{n}+\left(a_{1} z+b_{1} \bar{z}\right) d z^{n-1} d \bar{z}+\cdots+\left(a_{n} z+b_{n} \bar{z}\right) d \bar{z}^{n} .
$$

If $\omega_{1}$ is totally real and $p$ is an isolated singular point, by Theorem 4.1, $p$ is an isolated zero of the linear function $a_{0} z+b_{0} \bar{z}$ and hence, such linear function is regular. Since it is the linear part of the function $A_{0}, p$ is a regular point of $A_{0}$. Thus, $\operatorname{deg}\left(A_{0}, p\right)= \pm 1$ and $\operatorname{ind}(\omega, p)= \pm 1 / n$.

\section{Non degenerate differential forms}

Let $\omega$ be a totally real differential $n$-form on some open subset $U \subset$ $\mathbb{C}$ and let $p \in U$ be an isolated singular point. We can extend the notation introduced in Section 4 and denote by $\omega_{k}$ the homogeneous part of degree $k$ of $\omega$. That is, each one of the coefficients $A_{j}$ admits a Taylor expansion at $p$ and $\omega_{k}$ is the $n$-form whose coefficients are the homogeneous parts of degree $k$ in the expansion of the $A_{j}$. 
Definition 5.1. We say that $\omega$ is semi-homogeneous at $p$ if there is $k \geq 1$ such that $\omega_{i}=0$ for $i=1, \ldots, k-1$ and $\omega_{k}$ is a totally real differential $n$-form having $p$ as an isolated singular point. Note that when $k=1$, this is equal to the definition of simple singular point.

Assume for simplicity that $p=0$ and let

$$
\omega_{k}=A_{0}^{k} d z^{n}+A_{1}^{k} d z^{n-1} d \bar{z}+\cdots+A_{n}^{k} d \bar{z}^{n},
$$

where $A_{i}^{k}$ are homogeneous polynomials of degree $k$. We define the characteristic polynomial of $\omega$ as the (real) homogeneous polynomial of degree $k+n$

$$
P_{\omega}=A_{0}^{k} z^{n}+A_{1}^{k} z^{n-1} \bar{z}+\cdots+A_{n}^{k} \bar{z}^{n} .
$$

Let us denote by $\pi: \mathbb{R}^{2} \rightarrow \mathbb{C}$ the polar blow-up, that is, $\pi(r, t)=r e^{i t}$. We fix $\delta>0$ small enough such that $\pi((-\delta, \delta) \times \mathbb{R}) \subset U$ and $p=0$ is the only singular point of $\omega$ in such set.

Lemma 5.2. If $\omega$ is semi-homogeneous with principal part $\omega_{k}$, then

$$
\tilde{\omega}(r, t)= \begin{cases}\frac{1}{r^{k}} \omega\left(r e^{i t}\right), & \text { if } r \neq 0, \\ \omega_{k}\left(e^{i t}\right), & \text { if } r=0,\end{cases}
$$

defines a totally real differential $n$-form along $\pi$ on $(-\delta, \delta) \times \mathbb{R}$.

Proof: Suppose that $\omega$ is given by

$$
\omega=A_{0} d z^{n}+A_{1} d z^{n-1} d \bar{z}+\cdots+A_{n} d \bar{z}^{n}
$$

and let us denote by $A_{j}^{k}$ the homogeneous part of degree $k$ of $A_{j}$. By the Hadamard Lemma it follows that

$$
A_{j}\left(r e^{i t}\right)=r^{k} B_{j}(r, t)
$$

for some differentiable functions $B_{j}:(-\delta, \delta) \times \mathbb{R} \rightarrow \mathbb{R}$ such that $B_{j}(0, t)=$ $A_{j}^{k}\left(e^{i t}\right)$. In particular

$$
\tilde{\omega}(r, t)=B_{0}(r, t) d z^{n}+B_{1}(r, t) d z^{n-1} d \bar{z}+\cdots+B_{n}(r, t) d \bar{z}^{n} .
$$

As a consequence of the Lemma 5.2, if $\omega$ is semi-homogeneous, we can choose $n$ unit vector fields $X_{1}(r, t), \ldots, X_{n}(r, t)$ along $\pi$ on $(-\delta, \delta) \times \mathbb{R}$ which are pairwise linearly independent and solution of $\tilde{\omega}(r, t)(X)=0$. 
Moreover, we denote by $\theta_{j}(r, t)$ a differentiable determination of the angle of each vector field $X_{j}(r, t)$. Then we showed in the proof of Theorem 4.1, that it is possible to factor $\tilde{\omega}$ as

$$
\tilde{\omega}=f \lambda_{1} \ldots \lambda_{n},
$$

where $\lambda_{j}$ are the linear forms given by

$$
\lambda_{j}=e^{i \phi_{j}} d z+e^{-i \phi_{j}} d \bar{z},
$$

with $\phi_{j}=\pi / 2-\theta_{j}$ and $f:(-\delta, \delta) \times \mathbb{R} \rightarrow \mathbb{R}$ a non vanishing function.

Definition 5.3. The pull-back through $\pi$ of the $n$-form $\tilde{\omega}$ defines an $n$-form $\pi^{*} \tilde{\omega}$ on $(-\delta, \delta) \times \mathbb{R}$, which is called the polar $n$-form of $\omega$. Analogously, we call linear polar forms of $\omega$ the linear forms $\pi^{*} \lambda_{1}, \ldots, \pi^{*} \lambda_{n}$, in such a way that

$$
\pi^{*} \tilde{\omega}=f \pi^{*} \lambda_{1} \ldots \pi^{*} \lambda_{n}
$$

An easy computation gives

$$
\pi^{*} \lambda_{j}=2\left(\cos \varphi_{j} d r-r \sin \varphi_{j} d t\right),
$$

for each $j=1, \ldots, n$, where $\varphi_{j}=\phi_{j}+t$. Thus, each one of these polar linear forms has singular points $(0, t)$ with $\varphi_{j}(0, t)=\pi / 2+q \pi, q \in \mathbb{Z}$.

Note that a point $(0, t)$ can be a singular point of only one of the polar linear forms. In fact, suppose that

$$
\varphi_{j_{1}}(0, t)=\pi / 2+q_{1} \pi, \quad \varphi_{j_{2}}(0, t)=\pi / 2+q_{2} \pi,
$$

for some $q_{1}, q_{2} \in \mathbb{Z}$. Then

$$
\theta_{j_{1}}(0, t)-\theta_{j_{2}}(0, t)=\left(q_{2}-q_{1}\right) \pi,
$$

which implies that the corresponding vector fields are linearly dependent and hence, $j_{1}=j_{2}$.

Moreover, under some conditions it is possible to determine the topological type of these singular points. Let $\Lambda_{j}$ be the vector field given by

$$
\Lambda_{j}=r \sin \varphi_{j} \frac{\partial}{\partial r}+\cos \varphi_{j} \frac{\partial}{\partial t} .
$$

Then, the jacobian matrix at a singular point is

$$
D \Lambda_{j}(0, t)= \pm\left(\begin{array}{ll}
1 & -\frac{\partial \varphi_{j}}{\partial r} \\
0 & -\frac{\partial \varphi_{j}}{\partial t}
\end{array}\right),
$$

with eigenvalues $1,-\frac{\partial \varphi_{j}}{\partial t}$. As a consequence, $(0, t)$ is a hyperbolic singular point of $\pi^{*} \lambda_{j}$ if and only if $\frac{\partial \varphi_{j}}{\partial t} \neq 0$. Moreover, $(0, t)$ is of saddle type when $\frac{\partial \varphi_{j}}{\partial t}>0$ and of node type when $\frac{\partial \varphi_{j}}{\partial t}<0$. 
Lemma 5.4. Let $\omega$ be a semi-homogeneous totally real differential $n$-form and $p=0$ an isolated singular point. Then $z=e^{i t}$ is a root of the characteristic polynomial $P_{\omega}$ if and only if $(0, t)$ is a singular point of one of its polar linear forms. Moreover, it is a simple root if and only if $(0, t)$ is a hyperbolic singular point of such polar linear form.

Proof: In general, we have $\pi^{*} d z=e^{i t}(d r+i r d t)$ and $\pi^{*} d \bar{z}=e^{-i t}(d r-$ ir $d t$ ). In particular, when restricted to $r=0$, we get

$$
\pi^{*} \tilde{\omega}(0, t)=\left(\sum_{j=0}^{n} A_{j}^{k}\left(e^{i t}\right)\left(e^{i t}\right)^{j}\left(e^{-i t}\right)^{n-j}\right) d r^{n}=P_{\omega}\left(e^{i t}\right) d r^{n} .
$$

By using the factor of $\pi^{*} \tilde{\omega}$ in the polar linear forms, we see that

$$
\pi^{*} \tilde{\omega}(0, t)=f(0, t) \cos \varphi_{1}(0, t) \ldots \cos \varphi_{n}(0, t) d r^{n},
$$

which implies that

$$
P_{\omega}\left(e^{i t}\right)=2^{n} f(0, t) \cos \varphi_{1}(0, t) \ldots \cos \varphi_{n}(0, t) .
$$

Thus, $z=e^{i t}$ is a root of $P_{\omega}$ if and only if $(0, t)$ is a singular point of one of the polar linear forms.

Moreover, since $P_{\omega}$ is a homogeneous polynomial it is easy to check that $z$ is a simple root if and only if $\frac{d}{d t}\left(P_{\omega}\left(e^{i t}\right)\right) \neq 0$. But if we differentiate in the above expression, we arrive to

$$
\frac{d}{d t}\left(P_{\omega}\left(e^{i t}\right)\right)= \pm 2^{n} f(0, t) \frac{\partial \varphi_{j}}{\partial t}(0, t) .
$$

Therefore, it is a simple root if and only if $(0, t)$ is a hyperbolic singular point, by the remark after Definition 5.3.

Remark 5.5. Suppose that $z=e^{i t}$ is a root of the characteristic polynomial $P_{\omega}$. By the Lemma $5.4,(0, t)$ is a singular point of one the polar linear forms, that is, $\varphi_{j}(0, t)=\pi / 2+q \pi$, for some $j \in\{1, \ldots, n\}$, and $q \in \mathbb{Z}$. For each $p \in \mathbb{Z}, e^{i(t+p \pi)}= \pm z$ is also a root of $P_{\omega}$ and hence, there are $j_{p} \in\{1, \ldots, n\}$, and $q_{p} \in \mathbb{Z}$ such that $\varphi_{j_{p}}(0, t+p \pi)=\pi / 2+q_{p} \pi$. This implies that

$$
\varphi_{j}(0, t)-\varphi_{j_{p}}(0, t+p \pi)=\left(p-q_{p}\right) \pi,
$$


for any $p \in \mathbb{Z}$. But looking at the way the functions $\varphi_{j}$ are constructed, if this is true for some point $t \in \mathbb{R}$, then it must be true for any $t \in \mathbb{R}$. Then, by taking derivatives with respect to $t$,

$$
\frac{\partial \varphi_{j}}{\partial t}(0, t)=\frac{\partial \varphi_{j_{p}}}{\partial t}(0, t+p \pi)
$$

Thus, $(0, t)$ is a singular point of $\pi^{*} \lambda_{j}$ of saddle or node type if and only if $(0, t+p \pi)$ is a singular point of $\pi^{*} \lambda_{j_{p}}$ of saddle or node type respectively. In conclusion, the singularity type only depends on the direction determined by $z=e^{i t}$.

Definition 5.6. Let $\omega$ be a totally real differential $n$-form with an isolated singular point $p$. We say that $\omega$ is non degenerate at $p$ if it is semi-homogeneous and the characteristic polynomial has only simple roots.

Theorem 5.7. Let $\omega$ be a totally real differential $n$-form with a non degenerate singular point $p$. Then,

$$
\operatorname{ind}(\omega, p)=1-\frac{S^{+}-S^{-}}{n}
$$

where $S^{+}$and $S^{-}$denote the numbers of characteristic directions of saddle and node type respectively.

Proof: Denote by $S_{j}^{+}$and $S_{j}^{-}$the numbers of singular points of saddle and node type respectively of the polar linear form $\pi^{*} \lambda_{j}$ in the interval $[0,2 \pi n)$. Then,

$$
\sum_{j=1}^{n} S_{j}^{+}=2 n S^{+}, \quad \sum_{j=1}^{n} S_{j}^{-}=2 n S^{-} .
$$

Recall that such points are given by the points $(0, t)$ such that $\varphi_{j}(0, t)=$ $\pi / 2+q \pi$, with $q \in \mathbb{Z}$. Moreover, it is of saddle type when $\varphi_{j}$ is increasing at such point and of node type when it is decreasing. This implies that

$$
\varphi_{j}(0,2 \pi n)-\varphi_{j}(0,0)=\pi\left(S_{j}^{+}-S_{j}^{-}\right),
$$

for all $j=1, \ldots, n$. 
Now, by Corollary 4.3,

$$
\begin{aligned}
\operatorname{ind}(\omega, p) & =\frac{1}{n} \sum_{j=1}^{n} \frac{\theta_{j}(0,2 \pi n)-\theta_{j}(0,0)}{2 \pi n} \\
& =-\frac{1}{n} \sum_{j=1}^{n} \frac{\phi_{j}(0,2 \pi n)-\phi_{j}(0,0)}{2 \pi n} \\
& =-\frac{1}{n} \sum_{j=1}^{n} \frac{\varphi_{j}(0,2 \pi n)-2 \pi n-\varphi_{j}(0,0)}{2 \pi n} \\
& =1-\frac{1}{n} \sum_{j=1}^{n} \frac{\varphi_{j}(0,2 \pi n)-\varphi_{j}(0,0)}{2 \pi n} \\
& =1-\frac{1}{n} \sum_{j=1}^{n} \frac{S_{j}^{+}-S_{j}^{-}}{2 n}=1-\frac{S^{+}-S^{-}}{n},
\end{aligned}
$$

since $\phi_{j}(0, t)=\frac{\pi}{2}-\theta_{j}(0, t)$ and $\varphi_{j}(0, t)=\phi_{j}(0, t)+t$.

Definition 5.8. Let $\omega$ be a totally real differential $n$-form with a non degenerate singular point $p$. Given a characteristic direction of saddle type, we define a characteristic curve as the blow-down of a stable/unstable manifold. If the characteristic direction is of node type, then we choose a characteristic curve as one of the integral curves whose limiting tangent direction is the characteristic direction. By a sector we mean each one of the regions bounded by two consecutive characteristic curves $S_{1}$ and $S_{2}$. We say a sector is

(1) hyperbolic: if both $S_{1}$ and $S_{2}$ are of saddle type;

(2) parabolic: if $S_{1}$ is of saddle type and $S_{2}$ is of node type, or vice versa;

(3) elliptic: if both $S_{1}$ and $S_{2}$ are of node type.

Let $S^{+}$and $S^{-}$denote the number of characteristic directions of saddle and node type respectively and let $h$ and $e$ denote the numbers of hyperbolic and elliptic sectors respectively, then $e-h=2\left(S^{-}-S^{+}\right)$. Thus, we get the following immediate consequence of Theorem 5.7, which generalizes the well known Bendixson formula for the index when $n=1$. 
Corollary 5.9. Let $\omega$ be a totally real differential $n$-form with a non degenerate singular point $p$. Then,

$$
\operatorname{ind}(\omega, p)=1+\frac{e-h}{2 n},
$$

where $e$ and $h$ are the numbers of elliptic and hyperbolic sectors respectively.

Remark 5.10. When $\omega$ has a non degenerate principal part, it is possible to improve the formula for the index given in Remark 3.3. Let $X_{1}(r, t), \ldots, X_{n}(r, t)$ be unit vector fields along $\pi$ on $(-\delta, \delta) \times \mathbb{R}$ which are pairwise linearly independent and solution of $\tilde{\omega}(r, t)(X)=0$. Moreover, we suppose that they are chosen so that

$$
\theta_{1}(0, t)<\theta_{2}(0, t)<\cdots<\theta_{n}(0, t)<\theta_{1}(0, t)+\pi,
$$

where $\theta_{j}(r, t)$ denotes the determination of the angle of each vector field $X_{j}(r, t)$. Note that for $r=0$, these vector fields are solution of an equation with homogeneous coefficients, which implies that

$$
\theta_{1}(0, \pi)=\theta_{i}(0,0)+\pi m_{i},
$$

for some $m_{i} \in \mathbb{Z}$ and $i \in\{1, \ldots, n\}$. Then, it follows that

$$
\operatorname{ind}(\omega, p)=m_{i}+\frac{i-1}{n} \text {. }
$$

\section{Phase portrait of non degenerate singular points}

In general, the foliations of an $n$-form can present very complicated configurations around a singular point. When $\omega$ has a non degenerate singular point, the $n$ foliations are obtained as the image of the integral curves of the polar linear forms through the polar blow-down. Moreover, since the characteristic polynomial has only simple roots, then the problem is simpler, because the polar linear forms only have singularities of saddle or node type.

Definition 6.1. Let $\omega$ be a totally real differential $n$-form with a non degenerate singular point $p$. Let $x$ be a point near $p$ and let $L$ be one of the $n$ leaves of the web passing through $x$. We say that $L$ is

(1) hyperbolic: if $p$ is not an accumulation point of $L$;

(2) parabolic: if $p$ is an accumulation point on just one side of $L$;

(3) elliptic: if $p$ is an accumulation point on both sides of $L$.

If the leaf $L$ is hyperbolic (respectively parabolic, elliptic), then it corresponds to an integral curve of one of the polar linear forms joining two saddles (respectively a saddle and a node, two nodes). In order to 
have a complete description of the configuration of $\omega$, we need to know how many sectors the leaf is going to pass through when connecting the two characteristic curves (Figure 1).

(1) Hyperbolic (saddle-saddle).
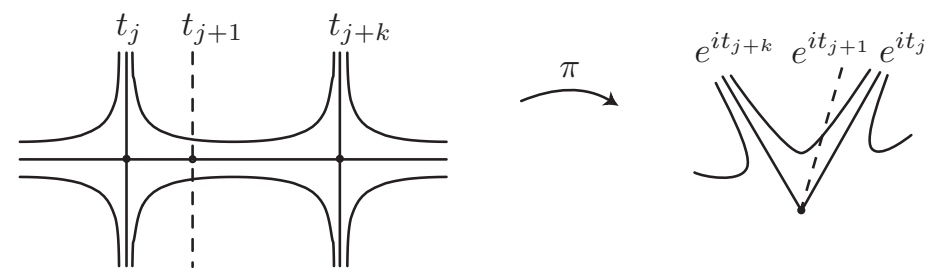

(2) Parabolic (saddle-node).
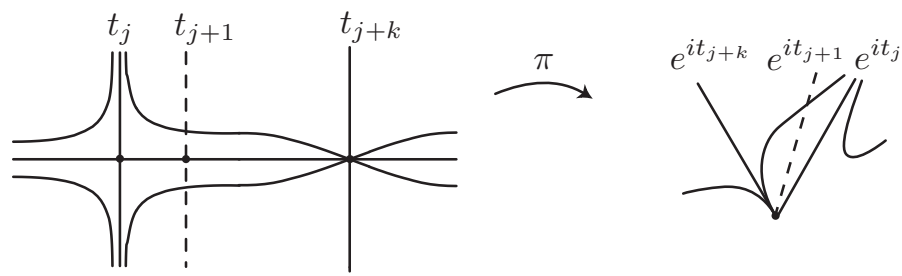

(3) Elliptic (node-node).
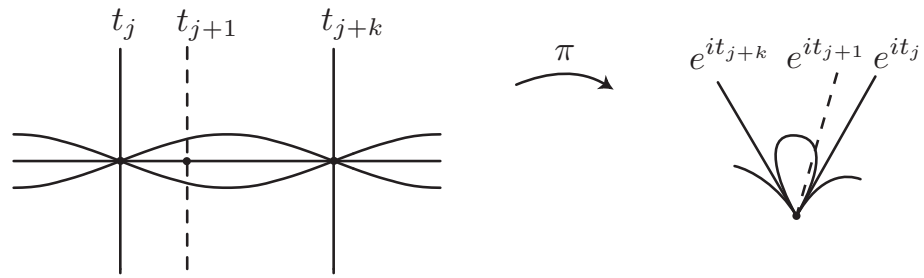

Figure 1. Types of leaves.

Lemma 6.2. Let $x$ be a point near $p$ and let $L$ be a hyperbolic leaf through $x$ joining two saddles. Assume that $L$ passes through $k$ sectors containing $n_{1}$ saddles and $n_{2}$ nodes (so that $n_{1}+n_{2}=k-1$ ). Then,

$$
k=n+2 n_{2} .
$$

Proof: Let $R$ be the union of the closed sectors that $L$ passes through, which is bounded by the two characteristic curves $S_{1}$ and $S_{2}$ of saddle type. Since $R$ is simply connected, we can separate the web in $R$ into 
$n$ foliations $\mathcal{F}_{1}, \ldots, \mathcal{F}_{n}$. We will assume that $L$ is a leaf of $\mathcal{F}_{1}$. Then $\mathcal{F}_{1}$ also contains the curves $S_{1}, S_{2}$ and all its other leaves of $\mathcal{F}_{1}$ are also hyperbolic.

Let $\mathcal{F}_{i}$ be one of the other foliations, with $i=2, \ldots, n$. We can use the leaves of $\mathcal{F}_{i}$ to define a continuous map $\phi_{i}: L \rightarrow S_{1} \cup S_{2}$. Given $y \in L$, we take the leaf $L_{i}$ of $\mathcal{F}_{i}$ passing through $y$. Because of transversality, either $L_{i}$ intersects $S_{1} \cup S_{2}$ in a single point which we define as $\phi_{i}(y)$ or $p$ is an accumulation point of $L_{i}$, in which case we define $\phi_{i}(y)=p$ (see Figure 2).

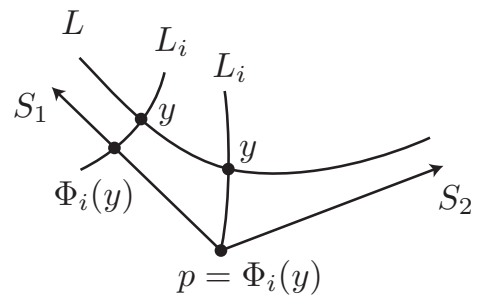

Figure 2. The map $\phi_{i}$.

Since the leaves of $\mathcal{F}_{i}$ are disjoint, we have two possibilities: either $\phi_{i}^{-1}(p)$ is just one point and $\mathcal{F}_{i}$ contains just one saddle, or $\phi_{i}^{-1}(p)$ is an interval, so that $\mathcal{F}_{i}$ contains one node and two saddles (see Figure 3 ).
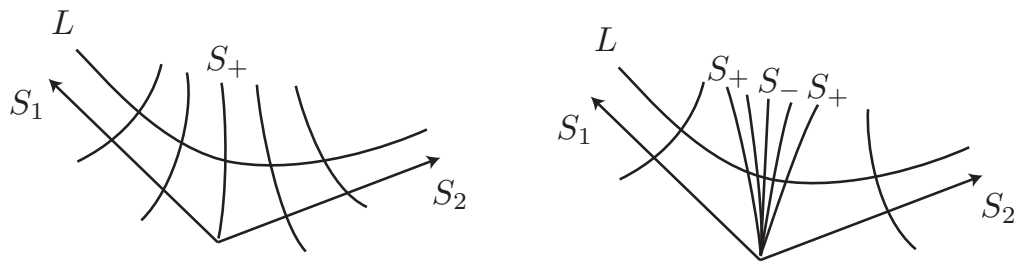

Figure 3. Possibilities for a hyperbolic leaf.

Finally, assume there are $a$ foliations of the first type and $b$ of the second type, with $a+b=n-1$. Then, $n_{1}=a+2 b$ and $n_{2}=b$, which gives the desired result.

Lemma 6.3. Let $x$ be a point near $p$ and let $L$ be an elliptic leaf through $x$ joining two nodes. Assume that $L$ passes through $k$ sectors containing $n_{1}$ saddles and $n_{2}$ nodes (so that $n_{1}+n_{2}=k-1$ ). Then,

$$
k=n+2 n_{1} .
$$


Proof: We assume that $p=0$ and that $\omega$ has the following principal part

$$
\omega_{k}=A_{0}^{k} d z^{n}+\cdots+A_{n}^{k} d \bar{z}^{n},
$$

where $A_{i}^{k}$ are homogeneous polynomials of degree $k$. We take now the inversion $z=1 / w$, which gives:

$$
d z^{n}=-\frac{d w}{w^{2 n}}-\frac{\bar{w}^{2 n} d w}{(w \bar{w})^{2 n}},
$$

and

$$
A_{i}^{k}(z)=A_{i}^{k}\left(\frac{1}{w}\right)=\frac{A_{i}^{k}(\bar{w})}{(w \bar{w})^{k}} .
$$

Then we obtain that in $\mathbb{C} \backslash\{0\}, \omega_{k}$ is equivalent to the differential form

$$
\sigma_{k}=A_{0}^{k}(\bar{w}) \bar{w}^{2 n} d w^{n}+\cdots+A_{n}^{k}(\bar{w}) w^{2 n} d \bar{w}^{n} .
$$

Note that $\sigma_{k}$ is also totally real with non degenerate principal part and the characteristic polynomial has the same roots as $\omega_{k}$, although the inversion transforms saddles into nodes and nodes into saddles. Moreover, elliptic leaves of the foliations of $\omega_{k}$ are transformed into hyperbolic leaves of $\sigma_{k}$ and vice versa. Thus, the result is a consequence of Lemma 6.2. In Figure 4 we present the result of taking the inversion of Figure 3.
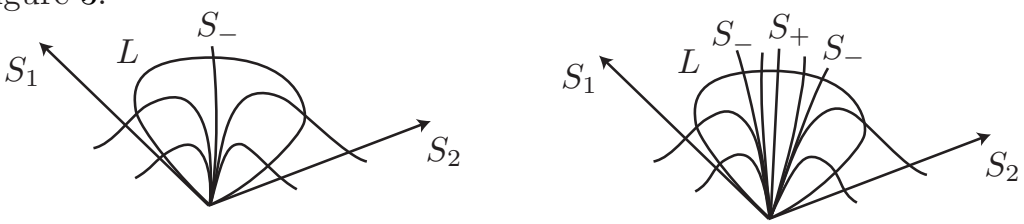

Figure 4. Possibilities for an elliptic leaf.

Lemma 6.4. Let $x$ be a point near $p$ and let $L$ be a parabolic leaf through $x$ connecting a saddle and a node. Assume that $L$ passes through $k$ sectors containing $n_{1}$ saddles and $n_{2}$ nodes (so that $n_{1}+n_{2}=k-1$ ). Then,

$$
k=1+2 n_{2} .
$$

Proof: We follow a similar argument to that of the proof of Lemma 6.2. We denote by $R$ the union of sectors containing the leaf $L$, which is bounded by the saddle $S_{1}$ and the node $S_{2}$. Let $\mathcal{F}_{1}, \ldots, \mathcal{F}_{n}$ be the $n$ foliations determined by $\omega$ in $R$ so that $L$ is a leaf of $\mathcal{F}_{1}$. 
For each one of the foliations $\mathcal{F}_{i}$, with $i=2, \ldots, n$ we have again two possibilities as listed in Figure 5. In one case $\mathcal{F}_{i}$ does not contain any characteristic direction, while in the other cased it contains one saddle and one node. If we denote by $a, b$ the number of foliations of each type respectively, we have $a+b=n-1$ and $n_{1}=n_{2}=b$. Therefore, we get $k=1+2 n_{2}$.
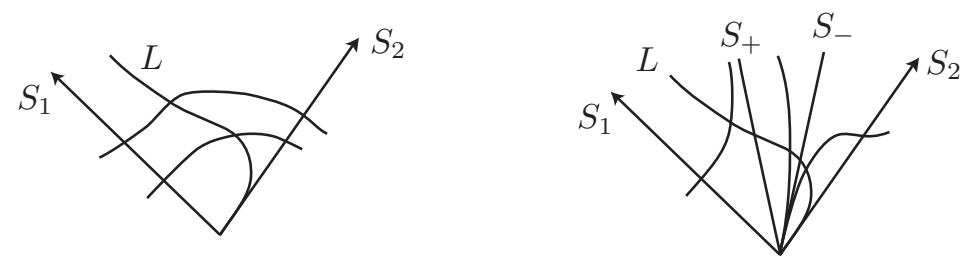

Figure 5. Possibilities for a parabolic leaf.

Remark 6.5. Once we know how many directions of saddle or of node type we have, as well as their relative position around the singular point $p$, the three above lemmas allow us to complete the phase portrait of all the leaves of the $n$ web determined by $\omega$. We call this the phase portrait of $\omega$ at $p$. When $n \leq 2$, it is well known that this is enough for topological classification, that is, if two differential $n$-forms have the same phase portrait at a point, then they are locally topologically equivalent. For $n \geq 3$, this is not true anymore because the curvature of the web is a topological invariant.

\section{Phase portraits near Darbouxian singular points}

In this section we give the possible phase portraits of Darbouxian singularities of totally real differential $n$-forms. These singularities are generic in the sense that a generic choice of coefficients in the linear part of the form should give a Darbouxian singularity.

Definition 7.1. We say that $p$ is a Darbouxian singular point of a totally real differential $n$-form $\omega$ if it is simple and the characteristic polynomial $P_{\omega}$ has only simple roots.

Theorem 7.2. Let $p$ be a Darbouxian singular point of a totally real differential $n$-form $\omega(n \geq 2)$. Then, there are only three possible phase portraits of the foliations of $\omega$ around $p$ : 
(1) Type $D_{1}$ (lemon): there are $n-1$ directions of saddle type with hyperbolic leaves passing through $n$ sectors.

(2) Type $D_{2}$ (monstar): there are $n$ directions of saddle type and one of node type; the hyperbolic leaves pass through $n+2$ sectors, while the parabolic leaves pass through one sector.

(3) Type $D_{3}$ (star): there are $n+1$ directions of saddle type with hyperbolic leaves passing through $n$ sectors.

Proof: Let $S^{+}$and $S^{-}$be the numbers of directions of saddle and node type respectively. The sum $S^{+}+S^{-}$is the total number of roots of the characteristic polynomial $P_{\omega}$, which has degree $n+1$. Since the roots are simple,

$$
0 \leq S^{+}+S^{-} \leq n+1, \quad S^{+}+S^{-} \equiv n+1 \quad \bmod 2 .
$$

Assume that $S^{+}+S^{-}=n+1$. If $S^{-} \geq 2$, then $S^{+} \leq n-1$ and by Theorem 5.7,

$$
\operatorname{ind}(\omega, p)=1-\frac{S^{+}-S^{-}}{n} \geq 1-\frac{n-1-2}{n}=\frac{3}{n} .
$$

This is not possible, by Corollary 4.5, since the index can only be $\pm 1 / n$. Thus, the only possibilities are $S^{+}=n+1, S^{-}=0$ or $S^{+}=n, S^{-}=1$ which correspond to the types $D_{3}$ and $D_{2}$ respectively. Note that the index in each case is $-1 / n$ or $1 / n$ respectively.

Next case is $S^{+}+S^{-}=n-1$. As above, if we suppose that $S^{-} \geq 1$, then $S^{+} \leq n-2$ and hence,

$$
\operatorname{ind}(\omega, p)=1-\frac{S^{+}-S^{-}}{n} \geq 1-\frac{n-2-1}{n}=\frac{3}{n} .
$$

The only possibility is $S^{+}=n-1, S^{-}=0$ which correspond to the type $D_{1}$ and has index $1 / n$.

Finally, assume that $S^{+}+S^{-} \leq n-3$. Then necessarily $S^{-} \geq 0$, $S^{+} \leq n-3$ and hence,

$$
\operatorname{ind}(\omega, p)=1-\frac{S^{+}-S^{-}}{n} \geq 1-\frac{n-3-0}{n}=\frac{3}{n} .
$$

The discussion about the number of sectors of hyperbolic or parabolic leaves is a consequence of above lemmas.

The above classification in the case $n=2$ gives the classification obtained by Darboux for the curvature lines around generic umbilic points of an immersed surface in $\mathbb{R}^{3}$ (see $[\mathbf{1}]$ and $[\mathbf{1 9}]$ ). A proof for the general case of Darbouxian singular points of quadratic forms can be found in $[\mathbf{1 1}]$. 


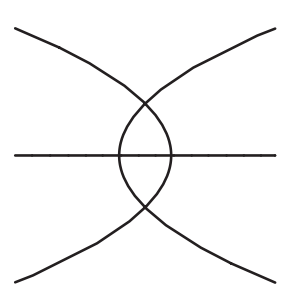

Quadratic lemon

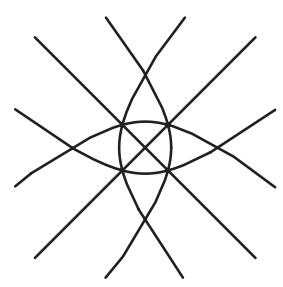

Cubic lemon

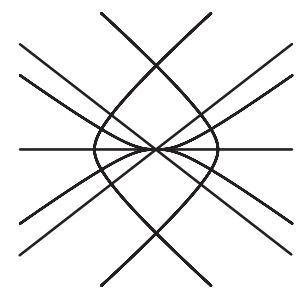

Quadratic monstar

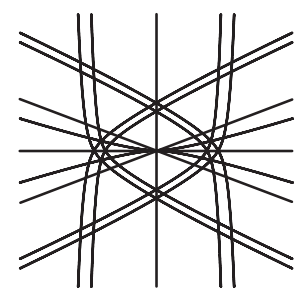

Cubic monstar

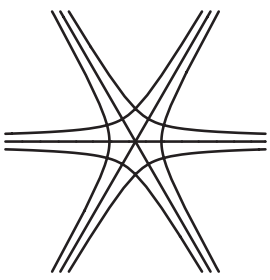

Quadratic star

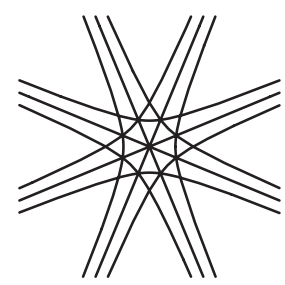

Cubic star

Figure 6. Pictures of the Darbouxian singularities $D_{1}$, $D_{2}, D_{3}$.

Example 7.3. Consider $\omega_{1}=\bar{z} d z^{n}+z d \bar{z}^{n}$. By Theorem 4.3,

$$
\operatorname{ind}\left(\omega_{1}, 0\right)=-\operatorname{deg}(\bar{z}, 0) / n=1 / n .
$$

Moreover, the characteristic polynomial is

$$
P_{\omega_{1}}=\bar{z} z^{n}+z \bar{z}^{n}=\bar{z} z\left(z^{n-1}+\bar{z}^{n-1}\right),
$$

which has $n-1$ real simple roots. Thus, for any $n, \omega_{1}$ has a Darbouxian singular point of type $D_{1}$ (lemon).

Now, let $\omega_{2, \epsilon}=(i z-(1+\epsilon) i \bar{z}) d z^{n}+(-i \bar{z}+(1+\epsilon) i z) d \bar{z}^{n}$, with $\epsilon>0$. In this case, the index is again $1 / n$ and the characteristic polynomial is

$$
\begin{aligned}
P_{\omega_{2, \epsilon}} & =(i z-(1+\epsilon) i \bar{z}) z^{n}+(-i \bar{z}+(1+\epsilon) i z) \bar{z}^{n} \\
& =(i z-i \bar{z})\left(z^{n}+\bar{z}^{n}\right)+\epsilon i z \bar{z}\left(z^{n-1}-\bar{z}^{n-1}\right) .
\end{aligned}
$$

Given $n$, it follows that for $\epsilon$ small enough, $P_{\omega_{2, \epsilon}}$ has exactly $n+1$ real simple roots. Then, $\omega_{2, \epsilon}$ has a Darbouxian singular point of type $D_{2}$ (monstar). 
Finally, we consider $\omega_{3}=z d z^{n}+\bar{z} d \bar{z}^{n}$. The index is now $-1 / n$ and $P_{\omega_{3}}=z^{n+1}+\bar{z}^{n+1}$. For any $n$, it has $n+1$ simple real roots, so that $\omega_{3}$ is of type $D_{3}$ (star).

Figure 6 gives the configurations of the foliations for the three examples $D_{1}, D_{2}, D_{3}$ in the cases $n=2$ (top) and $n=3$ (bottom) obtained with Mathematica $\left(D_{1}\right.$ and $\left.D_{3}\right)$ and with the program Homogeneous equations lines by A. Montesinos $[\mathbf{1 7}]\left(D_{2}\right.$ with $\left.\epsilon=1 / 2\right)$.

\section{References}

[1] J. W. Bruce And D. L. Fidal, On binary differential equations and umbilics, Proc. Roy. Soc. Edinburgh Sect. A 111(1-2) (1989), 147-168.

[2] J. W. Bruce and F. TARi, On binary differential equations, Nonlinearity 8(2) (1995), 255-271. DOI: 10.1088/0951-7715/8/2/008.

[3] M. Brunella and M. Miari, Topological equivalence of a plane vector field with its principal part defined through Newton polyhedra, J. Differential Equations 85(2) (1990), 338-366. DOI: 10.1016/0022-0396(90)90120-E.

[4] M. P. Do CARMo, "Differential geometry of curves and surfaces", Translated from the Portuguese. Prentice-Hall, Inc., Englewood Cliffs, N.J., 1976.

[5] G. Darboux, Sur la forme des lignes de courbure dans la voisinage d'un ombilic, Leçons sur la Theorie des Surfaces, IV, Note 7, Gauthier Villars, Paris (1896).

[6] A. A. Davydov, "Qualitative theory of control systems", Translated from the Russian manuscript by V. M. Volosov, Translations of Mathematical Monographs 141, American Mathematical Society, Providence, RI, 1994.

[7] J.-P. Dufour, Rigidity of webs, in: "Web theory and related topics" (Toulouse, 1996), World Sci. Publ., River Edge, NJ, 2001, pp. 106-113. DOI : 10.1142/9789812794581_0005.

[8] T. Fukui AND J. J. NuÑo-BAllesteros, Isolated roundings and flattenings of submanifolds in Euclidean spaces, Tohoku Math. J. (2) 57(4) (2005), 469-503. DOI: 10.2748/tmj/1140727069.

[9] T. Fukui and J. J. Nuño-Ballesteros, Singular points of equiangular binary differential equations, Preprint.

[10] T. Fukui and J. J. Nuño-Ballesteros, Higher order contact lines of surfaces in Euclidean spaces, in preparation.

[11] V. Guíñez, Positive quadratic differential forms and foliations with singularities on surfaces, Trans. Amer. Math. Soc. 309(2) (1988), 477-502. DOI: $10.2307 / 2000921$. 
[12] C. Gutierrez, I. Guadalupe, R. Tribuzy, and V. Guíñez, Lines of curvature on surfaces immersed in $\mathbb{R}^{4}$, Bol. Soc. Brasil. Mat. (N.S.) 28(2) (1997), 233-251. DOI: 10.1007/BF01233393.

[13] C. Gutierrez, R. D. S. Oliveira, and M. A. Teixeira, Positive quadratic differential forms: topological equivalence through Newton polyhedra, J. Dyn. Control Syst. 12(4) (2006), 489-516. DOI: $10.1007 / \mathrm{s} 10883-006-0003-1$.

[14] P. Hartman And A. Wintner, On the singularities in nets of curves defined by differential equations, Amer. J. Math. 75 (1953), 277-297.

[15] H. Hopf, "Differential geometry in the large", Notes taken by Peter Lax and John Gray, With a preface by S. S. Chern, Lecture Notes in Mathematics 1000, Springer-Verlag, Berlin, 1983.

[16] J. A. Little, On singularities of submanifolds of higher dimensional Euclidean spaces, Ann. Mat. Pura Appl. (4) 83 (1969), 261-335. DOI: $10.1007 / \mathrm{BF} 02411172$.

[17] A. Montesinos-Amilibia, Homogeneous equations lines, computer program available at http://www.uv.es/montesin.

[18] I. R. Porteous, The normal singularities of a submanifold, $J$. Differential Geometry 5 (1971), 543-564.

[19] J. Sotomayor And C. Gutierrez, Structurally stable configurations of lines of principal curvature, in: "Bifurcation, ergodic theory and applications" (Dijon, 1981), Astérisque 98-99, Soc. Math. France, Paris, 1982, pp. 195-215.

T. Fukui:

Department of Mathematics

Faculty of Science

Saitama University

255 Shimo-Okubo

Urawa 338-8570

Japan

E-mail address: tfukui@rimath.saitama-u.ac.jp

J. J. Nuño-Ballesteros:

Departament de Geometria i Topologia

Universitat de València

Campus de Burjassot

46100 Burjassot (València)

Spain

E-mail address: nuno@uv.es

Primera versió rebuda el 5 d'octubre de 2010, darrera versió rebuda el 20 de juny de 2011. 